\title{
IMPACTO PSICO-SOCIO-EDUCATIVO DE LA TECNOCIENCIA EN UNA HUMANIDAD GLOBALIZADA
}

\author{
Psico-socio-educative impact of the technoscience \\ in a globalized bumanity
}

\section{Impact socio-éducatif de la technoscience dans une bumanité mondialisée}

\begin{abstract}
Ana Isabel Paramá Díaz*, Isabel Caballero Caballero*, Juan R. Coca*** y Juan Milton Jair ARAGÓN PALACIOS****

* GIR Trans-REAL Lab. Universidad de Valladolid. Facultad de Educación. Campus Universitario "Duques de Soria". Departamento de Didáctica de las Ciencias Experimentales, Sociales y de la Matemática. 42004 Soria. anaisabelparama@uva.es; isabelcaballero@dce.uva.es * GIR Trans-REAL Lab. Universidad de Valladolid. Facultad de Educación. Campus Universitario "Duques de Soria". Departamento de Sociología y Trabajo Social.42004 Soria.juancoca@soc.uva.es ** Benemérita Universidad Autónoma de Puebla. Instituto de Ciencias Sociales y Humanidades. Av. Don Juan de Palafox y Mendoza 208, Centro. 72000 Puebla, México.miltonaragon@gmail.com
\end{abstract}

Fecha de recepción: enero de 2016 Fecha de aceptación: marzo de 2016

RESUMEN

El texto presenta una serie de ejemplos paradigmáticos en los que el desarrollo tecnocientífico lleva implícita una transformación a nivel psico-socio-educativo. El objetivo es reflexionar sobre las posibilidades del impacto que tiene el operar del sistema tecnocientífico, en diferentes ámbitos del sistema educativo. El trabajo realizado 
pone de manifiesto la interrelación existente entre los sistemas tecnocientífico, social, psíquico y educativo. El problema con el que nos encontramos es que materializar estas ideas supone una gran transformación del proceso educativo actual, originando que los agentes implicados se resistan a la adquisición de este conocimiento. En caso contrario, el sistema educativo se vería profundamente alterado.

Palabras clave: tecnociencia; desarrollo; enseñanza; música; sueño; género.

\section{SUMMARY}

The paper presents a series of paradigmatic examples. In which technoscientific development, involves a transformation at the psycho-socio-educational level. The objective is to present the possibilities of the impact that has the operating system techno-scientific, in different areas of the educational system. The work shows the interrelationship between the techno-scientific, social, psychological and educational systems. The problem is that a great transformation of the current educational process is necessary to make real these ideas, causing the agents involved resist the acquisition of this knowledge. If so, the educational system would be profoundly altered.

Key words: technoscience; development; teaching; music; sleep; gender.

\section{SOMMAIRE}

Le texte présente une série d'exemples paradigmatiques où le développement techno-scientifique implique la transformation au niveau psycho-socio-éducatif. L'objectif est de réfléchir sur les possibilités d'impact du fonctionnement du système techno-scientifique dans les différents domaines du système éducatif. Le travail mettre en lumière l'interconnexion entre les systèmes techno-scientifique, sociaux, psychologique et éducatif. Le problème est que concrétiser les idées constitue une transformation majeure du processus d'éducation actuel, ce qui rend les agents impliqués résister à l'acquisition de ces connaissances.

Mots clés: technoscience; développement; enseignement; musique; sommeil; genre.

\section{INTRODUCCIÓN}

La ciencia, anteriormente, era observada como una disciplina o actividad intelectual diferenciada de la tecnología. Se concebía, como exclusividad de la ciencia, desarrollar un conocimiento descriptivo-explicativo de la realidad circundante. El objetivo del científico era, y sigue siendo, "conocer" y "entender" el mundo que le rodea, impelido por la curiosidad o por la necesidad de controlar algún elemento trascendente en su vida. En cambio, la tecnología se concibe como un saber 
ANA ISABEL PARAMÁ, ISABEL CABALLERO, JUAN R. COCA Y JUAN MILTON JAIR ARAGÓN

eminentemente práctico y con una clara finalidad (telos), centrada en el desarrollo de artefactos tecnológicos que permitan al tecnólogo y a la sociedad superar los límites que la animalidad impone al hombre. No vamos a entrar a debatir la diferenciación entre técnica y tecnología, remitimos al lector a los grandes pensadores que han trabajado este tema. Nos referimos a Ortega y Gasset, Heidegger, Mitcham, Ellul, entre otros. Con el paso de los años, ambas áreas (ciencia y tecnología) se fueron complementando y fueron confluyendo, rompiendo con esa concepción de áreas separadas.

En la actualidad, resulta difícil diferenciar claramente el operar de ambas. No puede haber ciencia sin la mediación de la tecnología y no puede haber tecnología sin los descubrimientos de la ciencia. No obstante, es posible seguir manteniendo cierta distinción operacional en función de las finalidades de ambas. En este sentido hay autores, como Miguel Ángel Quintanilla (2005) o Ana Cuevas (2009), que piensan que dicha distinción es necesaria sobre todo a la hora de realizar una correcta política científica o tecnológica. Creemos que están en lo cierto. Ahora bien, a nuestro juicio, la tecnociencia hace referencia al gran sistema en el que la ciencia y la tecnología conviven, se retroalimentan y co-evolucionan. Ello no quiere decir que ciencia y tecnología sean absolutamente indistinguibles y que operen de igual forma en cuanto a su sentido.

Esta relación de la ciencia con la tecnología ha ido tomando más fuerza con el paso de los años hasta que se produjo la "fusión" entre ambas, cada vez más íntima y profusa, emergiendo de esta manera la Tecnociencia, un híbrido que ha dado lugar a la revolución tecnocientífica. Un concepto ampliamente estudiado en la filosofía y sociología de la ciencia, así como en determinados ámbitos educativos. De ahí que haya sido muy utilizado en el mundo interdisciplinar de la CTS: Ciencia, Tecnología y Sociedad. Así que hoy en día resulta muy difícil separar Ciencia de Tecnología. La línea divisoria entre ambas disciplinas es cada vez más estrecha. Tal es así que hay autores que piensan que todo es Tecnociencia. Autores como Stork, Barret, Ladrière, Latour y Hottois, entre otros, apuntan una convergencia entre ciencia y tecnología, llegando incluso a cuestionar la existencia de barreras fronterizas entre ambas (Echeverría, 2003).

Hace algunos años en un texto, que se convirtió en referencia inexcusable, Javier Echeverría (1995) mostró que dentro de la tecnociencia existen cuatro contextos: educación (enseñanza y difusión), innovación (descubrimientos e invenciones), evaluación (valoración) y aplicación. Estos cuatro contextos no actúan de modo independiente, sino que interactúan entre sí, haciendo que se origine una estrecha relación entre ellos. De ahí que el avance de la tecnociencia dependerá de que todos operen en conjunto. Como se habrá podido comprobar, la ciencia es uno de los pilares fundamentales del desarrollo de esta tecnociencia, por ello sigue siendo necesario reflexionar sobre esta actividad humana y conocer su impacto psico-socio-educativo actual. En caso contrario, podríamos caer en una brecha enorme entre la actividad tecnocientífica y la educativa.

Partimos de la siguiente hipótesis. Es importante analizar el impacto que podrían tener las recientes investigaciones tecnocientíficas en el desarrollo de otros 
ámbitos de conocimiento. En este trabajo, concretamente, nos centraremos en la educación. De ahí que nuestro estudio se enmarque dentro de los denominados estudios sobre el futuro (Ogilvy, 2002).

En base a lo anteriormente expuesto, el objetivo del presente texto es desarrollar una reflexión teórica sobre las posibilidades (positivas y negativas) que el sistema tecnocientífico ofrece al educativo a través del impacto futuro del desarrollo tecnocientífico actual. Para ello, se presentan y analizan varios ejemplos paradigmáticos que se han ido aportando desde la tecnociencia de carácter experimental al sistema educativo (tecnociencia educativa), planteando algunos de los retos socioeducativos y los condicionantes que ello generaría. Dichos ejemplos los hemos elegido al considerar que son ilustrativos de las potencialidades psicosocio-educativas que genera la actividad tecnocientífica. La selección de los artículos ha sido establecida a raíz de las impresiones que nos han ido transmitiendo, de manera informal, los docentes con los que hemos ido hablando. En este sentido pretendemos, como no puede ser de otro modo, dar respuesta a la sociedad a través de los agentes educativos con los que mantenemos contacto.

\section{LA INTENCIONALIDAD DE LA TECNOLOGÍA Y DE LA CIENCIA: CONOCER Y MODIFICAR EL MUNDO}

Antes de entrar a desarrollar los objetivos de nuestro trabajo, es conveniente hacer una serie de aclaraciones previas importantes para poder comprender y contextualizar el ámbito epistémico en el que se va a desarrollar esta investigación teórica.

El término tecnociencia fue planteado en el mundo académico, por primera vez, por el francés Bruno Latour a principios de la década de los ochenta. La idea de este autor tenía una clara intencionalidad de facilitar el trabajo de los estudiosos de la ciencia y la tecnología. Es decir, con el fin de evitar la interminable expresión ciencia y tecnología, Latour plantea el concepto de tecnociencia. No obstante, Javier Echeverría nos dice que es difícil afirmar con rotundidad que Latour haya sido el primero en utilizar dicha expresión. Pero el mérito de Latour, en cuanto al uso del concepto, es que lo empleó académicamente e hizo que tuviese impacto en ciencias sociales y humanas. Ahora bien, Echeverría (2003) muestra que seguramente ha sido usado por muchas personas sin conocer la propuesta del propio Latour y, previsiblemente, en un sentido distinto al del francés.

Es por tal motivo que Javier Echeverría (2003) diferencia entre ciencia, tecnología, tecnociencia y megaciencia (big science). Las distinciones entre ellas están referidas, fundamentalmente, a su intencionalidad y a los valores inherentes a las mismas. A nuestro juicio, la tecnociencia hace referencia al sistema que genera la ciencia y la tecnología, el sistema tecnocientífico. Dentro de dicho sistema nos encontramos con la ciencia y la tecnología. Ambas se retroalimentan y se condicionan entre sí, pero es posible mantener la diferenciación entre ambas en relación a que una tiene un telos epistémico y la otra un telos artefactual. 
En la actualidad, nos encontramos en un mundo que depende de forma creciente de la ciencia y la tecnología. Los procesos de producción, las fuentes de alimentación, la medicina, la educación, la comunicación o el transporte son todos campos cuyo presente y futuro están fuertemente ligados al desarrollo tecnocientífico. La ciencia y la tecnología han contribuido de forma asombrosa a mejorar nuestras condiciones de vida, aumentando tanto la esperanza de vida como su calidad y transformando los modos de interacción humanos. Al mismo tiempo, sin embargo, han ocasionado problemas y riesgos que requieren un análisis serio y exhaustivo (Núñez-Jover, 2007). El aumento de la contaminación, el uso de sustancias tóxicas, el deterioro progresivo del medio ambiente, la desertización, el empobrecimiento de la flora y la fauna y los accidentes y enfermedades relacionados con la tecnología son una parte importante de estos riesgos. De ahí que los avances tecnológicos sean usados en casi todas las actividades y por casi todas las personas. La utilización de la tecnología conduce a grandes controversias que suelen llevar a la falsa conclusión de que la tecnología es corruptora y perjudicial en sí misma. La tecnología es creada para conseguir beneficios, para modificar la realidad, para mejorarla, pero, muchas veces, su verdadera función es mal entendida y se utiliza en actividades incorrectas como la fabricación de material bélico o el abuso que conlleva adicciones.

La tecnociencia constituye un sistema dual creado con la finalidad de alcanzar los fines que persigue el ser humano. De hecho, Larry Laudan (1986) afirma que la ciencia busca solucionar los problemas que le van surgiendo a la humanidad en su devenir sociohistórico. Aunque, si se piensa con detenimiento, ¿qué sistema no está creado para nuestro propio beneficio, directa o indirectamente? Ahora bien, como han mostrado numerosos eticistas de la ciencia, Briggle y Mitcham (2012), Echevarría (2002), Coca (2010), Queraltó (2003), Longino (1990), Allchin (1998), entre otros, la ciencia en su proceso resuelve problemas, pero genera constantemente otros.

En este sentido, se puede mostrar el irremediable proceso de ideologización (a través de una visión politizada de esta actividad). La imagen benefactora de la ciencia y la tecnología, originada a partir de la ilustración, ya no se debe dar por supuesta, ni sus practicantes pueden pretender mantener su estatuto tradicional en la sociedad. Pero la tecnociencia también tiene aplicaciones beneficiosas para la sociedad. Además de los riesgos, el desarrollo aporta nuevas formas de relación y nuevos valores. No podemos concluir que la tecnología sea buena, mala o neutra, dependerá de la responsabilidad en el uso y del análisis preventivo de las consecuencias antes de tomar las decisiones. Dado que, en el operar la tecnociencia, de inicio no hay un sentido de lo bueno y lo malo, eso se acopla del correlato comunicacional que proviene de la ética, el cual opera en y para las reglas de este sistema exclusivamente.

Así como la ética, la objetividad es otro correlato presente en la tecnociencia que permite mantener una neutralidad en su proceder, pero con cierta tendencia a los procesos de reducción del mundo personal. Esto tampoco significa que 


\section{2 \\ ANA ISABEL PARAMÁ, ISABEL CABALLERO, JUAN R. COCA Y JUAN MILTON JAIR ARAGÓN \\ IMPACTO PSICO-SOCIO-EDUCATIVO DE LA TECNOCIENCIA EN UNA HUMANIDAD GLOBALIZADA}

tengamos que negar el factor humano vinculado al progreso del sistema. La cuestión está (Paramá, Francisco y Fernández, 2014) en buscar enmarcar el progreso tecnocientífico en un ámbito humanístico y personalizante (Coca, 2010), a pesar del correlato que despersonaliza a la tecnociencia. De tal forma que estos correlatos implican algo más allá del desarrollo tecnológico, porque no es en la tecnología, sino su forma de uso, donde radica el sentido humano de la tecnociencia.

La tecnociencia, como hemos visto, presenta una dualidad entre soluciones y problemas. Ello ha hecho que exista diversidad de interpretaciones de la tecnociencia. De hecho, Gabriel Marcel (2003) mantiene profundas reservas ante la tecnociencia, ya que el ser humano al hacer mal uso de ella tiene la posibilidad de destruir tanto su propia existencia como la existencia de la Naturaleza. Por lo que hay que ser precavidos y tener presente que los elementos psicológicos condicionan el quehacer humano. De hecho, los estudiosos de los imaginarios sociales y de las representaciones sociales son muy conscientes de esto.

En esta ocasión, nosotros asumimos esta premisa y no vamos a ocuparnos de los elementos arracionales, imaginarios, subjetivos que tiene la tecnociencia y que traen consigo transformaciones en el sistema. La mención es simplemente para dejar constancia de ello, y de que no deben olvidarse, estos elementos como parte de la construcción de la realidad tecnocientífica. En este sentido, y desde una perspectiva sistémica (Luhmann, 1992), la relación (interpenetración) entre los distintos sistemas que operan funcionalmente para configurar el sistema educativo implicaría un estudio extenso. Por ello, en esta ocasión, nos ocuparemos de la interpenetración entre los sistemas tecnocientífico, social, psíquico y el educativo.

\section{EL CONTRASENTIDO DEL SISTEMA TECNOCIENTÍFICO}

Dado el proceso de interpenetración del sistema económico-empresarial y el político en la esfera tecnocientífica, cada vez es mayor la concepción de que la actividad tecnocientífica no consiste en el descubrimiento de la verdad y el conocimiento de la realidad. El sistema tecnocientífico se pone al servicio de los intereses económicos, políticos y sociales. Y, en cualquier caso, inevitablemente la tecnociencia está enfocada en un aumento de la competitividad y de la productividad. De tal manera que la investigación se llega a mediatizar con fines económicos o políticos, sin los cuales, poder conseguir financiación para iniciar o proseguir la investigación es prácticamente imposible porque la investigación científica y tecnológica está condicionada por los planteamientos políticos ("las políticas científicas y tecnológicas»), económicos (rentabilidad) y sociales (necesidades sociales en el sentido más amplio de las palabras). Ante este hecho, el interés social, económico o político de las temáticas a investigar es lo que finalmente hace que determinados proyectos, investigaciones, intereses puedan avanzar frente a otros que pueden ser relegados. Esto lleva implícito que los primeros sean aquellos considerados como "rentables», mientras que los segundos son aquellos no interesantes económicamente e incluso irrelevantes (Maiso, 2015). 
El hecho de que el sistema tecnocientífico esté tan interrelacionado con el económico-empresarial trae consigo que los proyectos de investigación tengan cada vez más un fuerte sesgo en su carácter aplicativo y económicamente condicionado. Esto vicia las líneas de investigación de los grupos de trabajo, «obligando» a los agentes tecnocientíficos a dirigir sus investigaciones hacia esos intereses externos a la propia tecnociencia. Por otro lado, también debemos ser muy conscientes de que la comunicación del sistema tecnocientífico se vincula con el pensamiento de Occidente y con características con cierto grado de etnocentrismo (Coca, Santasilia y Valero-Matas, 2012; Paramá y Rodrigo, 2015).

Ahora bien, pese a esto, se están produciendo también ciertas matizaciones en el progreso de esta actividad. Ello es debido a la influencia e incorporación que tiene a medida que se va expandiendo por las diferentes culturas, emergiendo, cada vez más, una tecnociencia intercultural. No obstante, estas diferencias culturales son enormemente reducidas puesto que la tecnociencia implica una producción del conocimiento global. Lo que hace que este sistema parezca que se está convirtiendo en un subsistema del sistema económico o el político, por acoplar en su estructura correlatos que pertenecen a estos sistemas. De hecho, las patentes, la transferencia de conocimiento, la consecución de proyectos de investigación, los contratos con empresas, los programas tecnocientificos nacionales e internacionales, etc., son las guías de actuación de los agentes tecnocientíficos. Por esta razón, la gestión del conocimiento tecnocientífico tiene cada vez mayor importancia, así como el marketing de este tipo de conocimiento y de las empresas e instituciones que lo desarrollan (Coca, 2011).

Ahora bien, con independencia de estos elementos negativos propios del actual sistema tecnocientífico, la tecnociencia se está convirtiendo en uno de los elementos fundamentales de cambio de buena parte de las sociedades. De hecho, como veremos en este trabajo, puede llegar a generar una gran alteración del sistema educativo.

Acabamos de ver cómo la tecnociencia presenta una doble cara que puede resultar paradójica e, incluso, ambivalente. El sistema tecnocientífico contiene elementos ideológicos, etnocéntricos y también elementos de fuerte condicionamiento economicista. No obstante, también se están desarrollando en su interior una serie de perspectivas de gran interés para el ámbito educativo ya que son expresión de una mayor apertura de su conocimiento e, incluso, del propio sistema. En este contexto consideramos que es posible mostrar cómo, desde una perspectiva fuertemente convencional, los nuevos descubrimientos tecnocientíficos también aportan luz a determinados aspectos educativos.

Pese a la importancia que los estudios sobre la denominada STEM (Science, technology, engineering and mathematics) están teniendo en la actualidad, nuestro ámbito de análisis no se enmarca en este contexto. Nuestra perspectiva está más próxima, tal y como hemos dicho, a la denominada inicialmente CTS. Ello es así puesto que la CTS contempla un elemento crítico, reformista e, incluso, más subversivo que las sTEM. Por esta razón, y pese a las posibles consideraciones que uno 


\section{ANA ISABEL PARAMÁ, ISABEL CABALLERO, JUAN R. COCA Y JUAN MILTON JAIR ARAGÓN IMPACTO PSICO-SOCIO-EDUCATIVO DE LA TECNOCIENCIA EN UNA HUMANIDAD GLOBALIZADA}

pueda tener al leer este trabajo, consideramos que nuestra perspectiva de análisis es otra. En otros estudios realizados por nuestro grupo de investigación hemos desarrollado este tipo de estudios y análisis que exceden el ámbito del presente trabajo de investigación.

\subsection{Tecnociencia, música y educación}

A medida que progresa la ciencia aumenta la expectativa de vida, por lo que se prevé que aumentará el envejecimiento de las poblaciones, fundamentalmente, las occidentales. Esto traerá consigo, siguiendo la reflexión de Paul Feyerabend (1986), un incremento en la potencial ideologización y la posible dominación empleando la actividad tecnocientífica como pseudojustificación para ello. Ahora bien, la tecnociencia y su progreso generan una serie de elementos positivos que es en lo que nos vamos a centrar en este trabajo para intentar estudiar sus potencialidades. En otra ocasión estudiaremos los elementos negativos de dichas investigaciones.

El progreso tecnocientífico y el subsiguiente incremento de nuestra esperanza de vida también trae consigo el aumento de las patologías asociadas a la edad, por ejemplo, los trastornos neurológicos. Estas enfermedades neurológicas provocan en las personas afectadas importantes problemas y discapacidades que repercuten en su vida cotidiana y en su relación con las demás personas. Así mismo, las familias de estas personas también se ven afectadas debido al impacto que estas enfermedades genera en el self de las mismas. Aquí vemos, de nuevo, esta doble cara del progreso tecnocientífico.

Demos un pequeño salto. Recientemente se ha visto que uno de los cuidados que implica una mejora en la rehabilitación de ciertos pacientes es la musicoterapia. Este tipo de terapia aporta la estimulación cognitiva necesaria para que se pueda reducir la velocidad de degeneración neuronal, el dolor, las preocupaciones y la ansiedad que subyacen a estas dolencias en enfermedades como la demencia, el Alzheimer y el Parkinson (Koelsch, 2015). Esto aporta un bienestar al paciente que de otra forma no tendría. Clark y Warren (2015) aportan un dato más y es que estos enfermos conservan sus recuerdos musicales, sea cual sea la fase en la que se encuentra la enfermedad, ello implica, a nivel psicosocial, el mantenimiento de ciertos elementos del self y, por tanto, se reducen también los problemas familiares generados por estas patologías.

Al igual que ésta, otras enfermedades, como el déficit de atención por hiperactividad, enfermedad de Parkinson, epilepsia, trastornos emocionales diversos, podrían tener en la música beneficios educacionales terapéuticos. Koelsch (2015) afirma que gracias al uso de la música para el tratamiento psicológico de enfermedades neuronales es posible regular nuestras propias emociones y los estados de ánimo de los pacientes a través de la evocación anamnésica de momentos previos de su vida afectiva pasada. Esto incluye reducir el dolor, las preocupaciones y la ansiedad a través del proceso denominado emociones evocadas por la música. 
Estos trabajos nos permiten afirmar que existe una relación entre la música usada en terapia y nuestras competencias relacionales. Sin embargo, aunque el actual sistema tecnocientífico hipertrofia y fomenta la especialización individualizada para la consecución de estos avances tecnocientíficos, ha sido necesaria la ruptura y separación entre diferentes disciplinas científicas y el desarrollo de un compromiso de trabajo conjunto. Lo que ejemplifica la necesidad de un entorno de trabajo transdisciplinar, porque incrementan, sustancialmente, el éxito en el quehacer tecnocientífico y el desarrollo de productos y estrategias que solucionen los nuevos problemas a los que la humanidad se va enfrentando.

Teniendo presente esto, podemos conducir nuestra reflexión respecto al impacto educativo que tiene este ejemplo hacia la consideración de los elementos positivos que pueden ser generados fruto de los avances tecnocientíficos. En este sentido, y pese a los problemas de envejecimiento poblacional inherentes al progreso tecnocientífico, este envejecimiento también ha generado una serie de problemas y sus pertinentes soluciones. La música es, precisamente, una de dichas soluciones ya que permite un mayor autoconocimiento y autocontrol, así como fomenta la relajación y la evocación de instantes con importancia emocional. La pregunta que nos suscita todo esto es: ¿cuál es la razón de que la música no se incorpore, de manera habitual, a los centros educativos? No tenemos respuesta para ello más allá de una especie de exceso de importancia del currículo y de los contenidos. Es decir, una cierta hipertrofia de los elementos positivistas del sistema educativo. En este sentido hay que recordar que la música empleada para relajar y tomar conciencia de sí tiene graves problemas de evaluación cuantitativa y, por tanto, podría generar la idea, dentro de ese marketing tecnocientífico al que hemos hecho mención, de que los centros educativos no están realizando correctamente su trabajo. Esto nos permite inferir, hipotéticamente, que la incorporación de elementos difícilmente cuantificables (aunque fuese a través de rúbricas de evaluación) tiene problemas de implantación en los centros educativos.

Sea como sea, lo que sí parece indiscutible es que la música aplicada en el desarrollo tecnocientífico educacional aportaría un gran número de beneficios educativos, psicológicos, sociales y personales. Ahora bien, también implicaría un cambio sustancial en las dinámicas de trabajo en los centros educativos. Dinámica que implica cierta transformación paradigmática y, que sospechamos, podría resultar difícil de asumir por la academia dominante. Por esta razón se hace necesario seguir investigando en procesos de implantación de nuevas estructuras, herramientas e instrumentos educativos -cuya aplicación aportaría una buena cantidad de beneficios potenciales- en los diferentes niveles de la educación.

\subsection{Tecnociencia, sueño y educación}

Otro ejemplo interesante y aclaratorio de los objetivos de análisis de nuestro trabajo lo encontramos en el conocimiento psico-socio-onírico, el cual también está generando un gran número de interesantes posibilidades actualmente. Ello es 


\section{6 \\ ANA ISABEL PARAMÁ, ISABEL CABALLERO, JUAN R. COCA Y JUAN MILTON JAIR ARAGÓN IMPACTO PSICO-SOCIO-EDUCATIVO DE LA TECNOCIENCIA EN UNA HUMANIDAD GLOBALIZADA}

debido a que vivimos en un mundo en el que se están sucediendo un sinfín de estímulos que alteran nuestra cognición y generan un mundo sumamente cambiante. Esta mudanza constante se une a un proceso de aceleración social permanente. Por estas razones nuestro cerebro tiene que estar en contante alerta, a pleno rendimiento y adaptarse con facilidad. Los avances tecnocientíficos que nos permiten conocer cómo se lleva a cabo este proceso de adaptación del cerebro a ese entorno tan cambiante que la humanidad ha generado son siempre bienvenidos.

En un trabajo publicado por Dumoulin et al. (2015), se muestra que durante la fase REM (Rapid Eye Movement) del sueño es cuando se produce un mayor incremento de la plasticidad cortical basada en la experiencia vivida previamente. Esto tiene unas fuertes implicaciones epistemológicas y psicoeducativas. Porque, como demuestra la citada investigación, nuestro conocimiento se produce en buena medida a través de procesos imaginarios y arracionales, medidos por la experiencia vivida, pero consolidados como conocimiento a través de los sueños. La fase REM del sueño resulta ser imprescindible en nuestro aprendizaje. El estudio sugiere que dicha fase fomenta la transformación y evolución de los patrones neurales desarrollados durante la experiencia diaria.

No sólo eso, gracias a los procesos oníricos, las personas consolidamos y afianzamos los nuevos conceptos que adquirimos, que, hasta el momento en el que se produce el sueño, se encontraban en nuestro sistema cerebral, concretamente en la memoria, de manera inestable. Por lo tanto, parecería obvio que la estandarización de un período de sueño después del aprendizaje facilitaría el afianzamiento del mismo. Ello implicaría, como veremos más adelante, un impacto educativo, si nos tomásemos como sociedad en serio este trabajo, pues los estudios científicos sobre sueño en educación no han tenido gran importancia.

En España, en el año 2008, la Asociación Española de Psiquiatría del Niño y el Adolescente (AEPNYA) emitió una serie de protocolos clínicos entre los que estaba el referido a los trastornos del sueño. En él se afirma que este tipo de trastornos son frecuentes en niños y adolescentes y un 20-30\% requieren intervención. La poca importancia que socialmente tiene el sueño trae consigo que en adolescentes sean comunes los patrones irregulares del sueño o el sueño deficiente. Según este protocolo, ello es debido a la adquisición de hábitos inadecuados durante la etapa escolar. De tal modo que trabajos como el de Velten-Schurian et al. (2010) se vuelven pertinentes sobre este problema, porque investigan la asociación entre los patrones de sueño con comportamientos sociales. Estos autores llegan a afirmar que la reducción del tiempo de sueño predice una mayor frecuencia en las conductas delictivas, así como un mayor número de problemas de concentración. Esto nos muestra la importancia que el sueño tiene en el desarrollo de las personas, principalmente, cuando son menores.

Paradójicamente, después de una búsqueda en distintas bases de datos, hemos podido comprobar que las investigaciones sobre el sueño se centran en el ámbito de las ciencias de la salud. Pareciera que los educadores, por lo menos en lo relativo a la investigación científica, se encuentran ajenos a los avances del 
conocimiento científico desarrollado en otras disciplinas del conocimiento. La otra posibilidad que nos viene a la cabeza reside en que los docentes consideren que la educación relativa a la higiene del sueño debe restringirse única y exclusivamente a los hogares. Estos aspectos deberían ser estudiados en posteriores trabajos para poder aclarar este aspecto. Así mismo debemos ser conscientes de las limitaciones que tiene el entorno educativo formal para poder establecer procesos de implantación de higiene del sueño en los centros. Ahora bien, esto no obsta para que la educación sobre la higiene del sueño pueda ser implantada en los centros educativos como elemento transversal.

Por otro lado, los trabajos relacionados con la evolución neuronal y la plasticidad cerebral, además de estar relacionados con una buena higiene del sueño, también están vinculados a los nuevos procesos y a las innovaciones educativas. En este sentido, el trabajo de Dumoulin et al. (2015) nos muestra que el cerebro tiene una amplia capacidad de adaptación y aprendizaje cuando nuestro entorno presenta cierto grado de variabilidad y complejidad.

El cerebro está constituido por neuronas, células interconectadas entre sí por unas estructuras denominadas sinapsis. Estas conexiones sinápticas no son estáticas, sino que tienen una propiedad fundamental, la plasticidad. Esta plasticidad permite que las neuronas cambien su distribución axonal conforme a sus necesidades internas y en respuesta a nuestra actividad externa. Es decir, existe capacidad para generar nuevas conexiones sinápticas entre las neuronas existentes e incrementar la comunicación entre ellas. Ello implica un buen número de consideraciones educativas.

Una de éstas es que, pese a que las rutinas son importantes en educación (Vallet, 2004; Zabalza, 2010), es necesario, si tenemos en cuenta el conocimiento tecnocientífico expuesto, que las rutinas sean mucho más dinámicas y menos estáticas que las establecidas actualmente. Así mismo, las dinámicas de trabajo y la propia aula en los centros educativos deberían ir cambiando para incrementar la plasticidad mental del niño. Las nuevas pedagogías de la cooperación, la flipped classroom, la gamificación, así como el uso de las TIC, entre otras, han aportado dinamismo a las aulas lo que, previsiblemente, traería consigo una mayor plasticidad en las mentes de los alumnos.

Ahora bien, sin un buen hábito de sueño serán poco eficaces los establecimientos efectivos de los procesos de enseñanza-aprendizaje en el aula. El dinamismo educativo se verá afectado por el previsible bajo rendimiento de los alumnos. Por ello, y quizá de manera irreverente, los responsables de los centros de educación deberían ir pensando si sería conveniente establecer zonas de sueño en las escuelas o incorporar la higiene del sueño como elemento transversal del currículum, fundamentalmente en la etapa de educación infantil. Con lo dicho, especialmente con el primer punto, la transformación de los colegios sería sustancial. Primero, al incrementarse la dinamicidad interna de los centros educativos. Segundo, la escuela dejaría de ser un lugar de mero aprendizaje, en el sentido tradicional, para convertirse en una zona de crecimiento y desarrollo personal. Recuérdese que en 
el primer ciclo de educación infantil es habitual que estas zonas existan y sean utilizadas habitualmente. En el segundo ciclo de educación infantil estos elementos han desaparecido de los centros educativos.

\subsection{Tecnociencia, género y educación}

Un tercer aspecto relevante para la consecución de los intereses de la investigación de este trabajo lo encontramos en los aspectos relacionados con los condicionantes de género presentes en el desarrollo de las ciencias. De hecho, si echamos un vistazo a lo largo de la historia de la ciencia y la tecnología, vemos que el número de mujeres que se citan es bajo, frente al elevado número de hombres que aparecen mencionados en la bibliografía. No obstante, existen diversas evidencias que ponen de manifiesto la importancia de las mujeres en determinados ámbitos científicos, de las que mencionaremos un par de ejemplos a continuación:

- La medicina fue practicada de forma informal por mujeres curanderas, comadronas o monjas en los conventos hasta la institucionalización de la misma, a partir del siglo XIII. El caso de la ginecología es especialmente revelador, ya que pasó de ser una actividad exclusiva de mujeres a una práctica médica y, por tanto, propia de hombres (Donnison, 1977; Ehrenreich y English, 1976).

- Los salones y academias científicas femeninas de los siglos XVII y XVIII. Excluidas de las academias científicas oficiales, muchas mujeres de clase alta y con intereses científicos dirigían salones en Francia en los que se discutían temas de actualidad y se promocionaba a jóvenes talentos sin medios. Las salonnières no podían entrar en las academias, pero mediaban e influían sobre los hombres que lo hacían. En Gran Bretaña, las mujeres organizaron academias alternativas y grupos de botánica, biología, astronomía, que realizaron importantes contribuciones, sobre todo, en tareas de colecta de datos (Alic, 1986; Schiebinger, 1989).

Trabajo invisible, no reconocido, que las mujeres realizan en la ciencia: maestras, ilustradoras, editoras, divulgadoras, técnicas de laboratorio, programadoras de informática. El papel de las mujeres ha sido, por ejemplo, valiosísimo en el nacimiento y desarrollo de determinadas áreas como la botánica, en las que realizaron el trabajo de recolección.

Estas evidencias han puesto de manifiesto que, si bien a lo largo de la historia la presencia de mujeres en las disciplinas científicas y en la tecnología ha sido inferior a la de los varones, su número no es tan pequeño como se suele afirmar. Sin embargo, su presencia, probablemente, quede oculta por prejuicios y concepciones caducas de lo que es la historia de la ciencia y la tecnología. Cuando se habla de mujeres y ciencia, la reacción inmediata es la de indicar la poca presencia de éstas en el desarrollo de la ciencia. Sólo once premios Nobel de física, ciencia 
y medicina se otorgaron en el siglo pasado a mujeres. Sin embargo, la historia de la ciencia relatada por mujeres ha descubierto muchas mujeres importantes dentro de este campo (Alic, 1986; Pérez Sedeño, 1994; Solsona y Pairó, 1997).

La historia de las mujeres tecnólogas tiene sus propios problemas y dificultades. Entre ellos estaba el ocultamiento sistemático de las mujeres que, en muchos casos, era promovido por la legislación sobre patentes en algunos países. Al no tener la mujer derecho de propiedad, son el padre o el marido o algún otro hombre los que aparecían en los registros de patentes como responsables de las invenciones hechas por mujeres. Por otra parte, las historias de la tecnología han pasado por alto el ámbito de lo privado, es decir, de lo femenino, en el que se utilizaban y utilizan tecnologías propias de las tareas tradicionalmente determinadas por la división sexual del trabajo, teniendo como consecuencia que inventos relacionados con la esfera de lo doméstico y la crianza, realizados por mujeres, no han contado como desarrollos «tecnológicos» (Pérez Sedeño, 1998b).

La historia pone de manifiesto cómo las oportunidades de las mujeres han variado con el tiempo y con las barreras estructurales e institucionales existentes desde el nacimiento de la ciencia moderna. Hoy en día la discriminación por razón de sexo no existe de un modo explícito en las instituciones científico-tecnológicas occidentales, pero esta abolición es muy reciente. Diversos trabajos en historia de la ciencia se han dedicado a rastrear a través del tiempo las ideas científicas sobre la naturaleza de las mujeres que contribuyeron a fundamentar su supuesta inferioridad y a mantenerlas alejadas de la producción de conocimiento y tecnología (Russet, 1989; Schiebinger, 1989; Tuana, 1993). La segregación institucional de la mujer fue desde siempre acompañada por teorías que pretendían sustentarla bajo una supuesta base científica. Las ideas sobre la inferioridad intelectual de la mujer se remontan hasta la antigüedad griega y en el siglo XVI se generalizó el debate sobre la educación de la mujer. La idea común era que la mujer es por naturaleza malvada, superficial, tonta y estúpida, lujuriosa e inconstante, poco apta, por lo tanto, para el estudio (Pérez Sedeño, 1998a).

A pesar del paso del tiempo, la ciencia contemporánea aún continúa empeñada en la tarea de identificar las diferencias sexuales en habilidades cognitivas. La búsqueda de diferencias en los cerebros masculinos y femeninos que expliquen y justifiquen la desigual presencia de hombres y mujeres en ciertos ámbitos científicos sigue siendo un importante programa de investigación en biología y psicología: estudios de dimorfismo sexual, análisis de los condicionamientos genéticos, hormonales y de estructura cerebral que ocasionan diferentes disposiciones en los dos sexos para distintas tareas. Así, por ejemplo, el estudio psicológico de las diferencias sexuales en habilidades cognitivas informa de una superioridad masculina en habilidad matemática y espacial, y una superioridad femenina en habilidades verbales. Estas diferencias explicarían el escaso número de mujeres en ingenierías o ciencias exactas, profesiones que requieren habilidad para las matemáticas y las relaciones espaciales. Un buen número de investigadoras han puesto de manifiesto los diversos tipos de sesgos que incorporan estos estudios, restando fuerza a la idea 
de que nuestros «cerebros diferentes» son la causa de las desigualdades existentes (González-García, 1998; Unger y Crawford, 1996).

Una muestra de la convergencia que se produce en los análisis de género entre el compromiso político y el análisis metacientífico son los múltiples intentos de participación directa en las prácticas que se construyen en la tecnociencia que llevan a cabo las mujeres. Lejos de limitarse a la mera función crítica, el análisis de género se enfrenta también a la difícil tarea de proponer alternativas constructivas para estructuras y contenidos de conocimiento científico insatisfactorios en las que se reconozca la ciencia como una práctica social. Los esfuerzos más notables se llevan a cabo en biología, psicología, antropología o sociología. En psicología, por ejemplo, diversas investigadoras feministas han propuesto nuevos métodos para la investigación psicológica y la psicoterapia que atiendan los rasgos contextuales de las situaciones, la complejidad del razonamiento de los sujetos y la relación entre el investigador/ terapeuta y su sujeto/paciente (Hare-Mustin y Marecek, 1994; Morawski, 1994).

Por otro lado, diversos estudios han puesto de manifiesto que las actitudes relacionadas con la ciencia dependen de diversos factores (Manassero-Mas y Vázquez-Alonso, 1996; Simpson y Oliver, 1990), entre los cuales el sexo de los estudiantes es uno de los más importantes (Fensham, 2004; Greenfield, 1996; Hendley, Stables y Stables, 1995; Jiménez y Álvarez, 1992; Lee y Bryk, 1986). En general, algunas actitudes relacionadas con la ciencia hecha por las mujeres son peores que las de los hombres, aunque el tamaño de las diferencias depende, a su vez, de factores muy diversos relacionados con la educación, la cultura o el país (SjØberg, 2005; Trumper, 2004).

A lo largo del siglo pasado, los países de la ocDE redujeron de forma importante las diferencias de género en muchas áreas de la educación y del empleo, incluyendo el rendimiento académico, el salario y la participación en el mercado laboral. Este hecho implica que la capacidad no conoce género. Con las mismas oportunidades, chicas y chicos, hombres y mujeres tienen (o deberían tener) las mismas posibilidades de alcanzar los niveles más altos.

Pero no debemos olvidar que existen otras diferencias de género en educación. Los chicos están menos implicados en su colegio o instituto que las chicas y obtienen rendimientos más bajos. También es más probable que dejen antes el instituto, muchas veces sin titulación. Y, por otra parte, en la universidad, las mujeres están menos representadas en áreas como matemáticas, física o informática.

En la mayoría de los países participantes en PISA, entre los alumnos de buen rendimiento, las chicas sacan peores resultados en matemáticas que los chicos y en el nivel alto, en ningún país lo hacen mejor. En general, las chicas tienen menos confianza en sí mismas que los chicos para resolver problemas matemáticos o científicos. Las chicas tienden también a reconocer un sentimiento de ansiedad hacia las matemáticas, y esto se observa incluso entre las chicas de mejor rendimiento escolar. De media, en los países de la OCDE, la diferencia en puntuación en matemáticas entre los chicos y chicas de mejor rendimiento es de 19 puntos. Sin embargo, si comparamos chicas y chicos que dijeron tener un nivel similar de 
confianza en sí mismos con respecto a las matemáticas, la brecha de género desaparece. Por otro lado, PISA revela que las chicas lo hacen mejor cuando se les pide una actividad de matemáticas o ciencias parecida a las que acostumbran a hacer en clase. Pero cuando se les pide que "piensen como científicas", las chicas lo hacen peor en comparación con los chicos, por ejemplo, al pedirles que formulen situaciones matemáticas. Los chicos también sacan mejores resultados cuando se les pide que apliquen su conocimiento científico a una situación dada, que interpreten fenómenos científicos y que hagan una predicción de los cambios. Esta diferencia de género podría relacionarse con la confianza que los alumnos tengan en sí mismos. Al tener más confianza, se dan más libertad para equivocarse, para practicar procesos de prueba y error que resultan fundamentales para adquirir mayor conocimiento y comprensión de las matemáticas o de las ciencias (OCDE, 2015).

Además, analizando los materiales escolares de ciencias, se puede constatar que se caracterizan por una ausencia casi total de imágenes de mujeres, o la presentación de éstas en los roles tradicionales «decorativos» o maternales, volviéndose su presencia más escasa a medida que se avanza en el nivel educativo (VázquezAlonso y Manassero-Mas, 2003; López-Navajas, 2014). Los programas de física se centran en las áreas tradicionalmente consideradas masculinas, como la mecánica, la electricidad o el magnetismo, prestando menos atención a áreas posiblemente más susceptibles de atraer a las alumnas, como la energía nuclear, los fenómenos meteorológicos o las aplicaciones de la física en medicina o arte.

En los últimos años se han realizado diversos trabajos sobre el papel de la mujer a lo largo de la historia de la ciencia y la transmisión de sus investigaciones a la población, en los cuales se ha puesto de manifiesto que las mujeres son prácticamente invisibles tanto en los libros como en Internet (García, 2013; López-Navajas, 2014). Así mismo, también se han planteado diversas propuestas para llevar a cabo en el aula con el objetivo de dar a conocer el importante papel que la mujer ha tenido en el desarrollo científico (Lires, Nuño y Solsona, 2003; Martínez, 2012).

Teniendo en cuenta todas las ideas expuestas, consideramos que la educación puede y debe ser un medio a utilizar para fomentar el interés de la tecnociencia entre nuestros jóvenes, y en especial entre las mujeres. Para ello, las distintas instituciones educativas deberían reconsiderar los contenidos curriculares, orientándolos hacia campos de interés de las niñas, a su experiencia personal y a proporcionarles modelos femeninos a seguir. Tenemos enfrente otro reto más para la tecnociencia, además de lo intercultural, se tiene que buscar la equidad de género en su operar, pues el desarrollo de ésta no depende de género, raza o credo, depende de la ruptura de sus paradigmas. Ahí hay un área importante en la formación de los futuros tecnocientíficos.

\section{ENSEÑANZA Y TECNOCIENCIA}

A pesar de que a un nivel social amplio como el periodístico o el político, y análogamente, en los medios académicos, se discute y apoya la necesidad del 
desarrollo del "conocimiento tecnológico", es poca la comprensión clara porque su significado usualmente se le asocia o identifica con los artefactos. Lo cual limita totalmente el rico significado epistemológico del concepto de tecnología y de la posibilidad de su integración en la enseñanza y didáctica.

Según Herrera (2009), el concepto de tecnología se puede representar por medio de una terna que contiene: (i) a la práctica tecnológica, es decir, el «sistema de actividades orientadas» mismo; (ii) al artefacto conceptual, es decir, el conocimiento necesario o por poseer, y (iii) al artefacto material (materia reorganizada para cumplir funciones). Los dos últimos son medios de producción y producto y también objeto de transformación de la actividad social orientada. Esta "actividad orientada" tiene ejecutores humanos en distintos niveles del proceso productivo, los cuales no actúan solos o aislados sino como componentes de sistemas concretos de transformación o tecnosistemas (Herrera, 1992). Donde la mayoría de las acciones se producen en cooperación, es decir, que la mayoría de las prácticas tecnológicas son "actividades socializadas» en las cuales se cumple con una "división de trabajo" o de "especialidad".

Para nosotros, esta idea es muy importante para la educación, pues su cumplimiento conduce al "trabajo en equipo" y remite al pensamiento sobre los efectos sociales y naturales de la transformación. Entendemos que este aprendizaje basado en la participación como orientador de las enseñanzas tecnocientíficas implica necesariamente un cambio en el papel que el docente tiene actualmente en el desarrollo curricular. Si es cierto que a participar se aprende participando, también resulta necesario haber participado para enseñar a participar. No cabe promover propuestas de enseñanza participativas que no cuenten con la participación y el compromiso de los docentes, que serán sus dinamizadores.

También es necesario poner de manifiesto que determinadas personas se sienten incapaces de controlar ciertos productos tecnológicos, o de afrontar simples razonamientos relacionados con la ciencia. La educación debería disminuir esa inseguridad que algunos ciudadanos tienen, de tal forma que se pudiera disfrutar de los crecientes beneficios de la era de la ciencia y la tecnología, garantizando, al mismo tiempo, la protección de la salud y el medio. Contribuyendo con el conocimiento a la toma de decisiones sobre el desarrollo científico y tecnológico en el que estamos inmersos, así como de sus consecuencias. Por lo tanto, la alfabetización científica será necesaria para contribuir a formar ciudadanos, y en su caso futuros científicos, que sepan desenvolverse en un mundo como el actual, que conozcan el importante papel que la ciencia desempeña en sus vidas personales y profesionales y en nuestra sociedad. Ciudadanos cuya formación les permita reflexionar y tomar decisiones apropiadas en temas relacionados con la ciencia y la tecnología (Aikenhead, 1985; Bingle y Gaskell, 1994; Gil, Carrascosa, Furió y Martínez-Torregrosa, 1991; Solbes y Vilches, 1997).

La alfabetización científica debe ser concebida como un proceso de «investigación orientada» que, superando el reduccionismo conceptual, permita a los alumnos participar en la aventura científica de enfrentarse a problemas relevantes y 
(re)construir los conocimientos científicos. Que habitualmente la enseñanza transmite ya elaborados, lo que favorece el aprendizaje más eficiente y significativo.

Una idea comúnmente aceptada por los diseñadores de currículos y los profesores de ciencias es que la educación científica ha estado, hasta hace relativamente poco tiempo, orientada para preparar a los estudiantes como si todos pretendieran llegar a ser especialistas en biología, física o química. Por ello, los currículos planteaban, como objetivos prioritarios, que los estudiantes supieran, fundamentalmente, los conceptos, principios y leyes de esas disciplinas. Dicha orientación ha sido modificada, ya que la educación científica se plantea ahora como parte de una educación general para todos.

Algunos investigadores parecen aceptar esta contraposición entre educación científica para todos y preparación de los futuros científicos. Así, Fensham (1988) o el mismo Bybee (1997) sostienen que en muchos aspectos los objetivos de enseñar ciencias para futuros científicos y para futuros ciudadanos son contradictorios, no complementarios. Un programa de ciencias para futuros científicos contemplaría la ciencia "desde dentro", mientras que el programa para futuros ciudadanos lo haría desde la perspectiva de la sociedad. Ello es lo que justifica el énfasis actual de los currículos en los aspectos sociales y personales (National Research Council, 1996), puesto que se trata de ayudar a la gran mayoría de la población a tomar conciencia de las complejas relaciones ciencia-sociedad, para permitirles participar en la toma de decisiones y, en definitiva, a considerar la ciencia como parte de la cultura de nuestro tiempo.

El hecho de que la ciencia y la tecnología estén omnipresentes en la vida actual y que el papel de sus protagonistas, los científicos y los ingenieros, esté reconocido en la vida social (aunque no siempre sea del todo percibido), seguramente, explica el escaso debate público sobre los contenidos que deben tener las enseñanzas de las ciencias y las tecnologías en los procesos de reformas educativas. En general, se acepta que es bueno que se enseñen más contenidos de matemáticas, de ciencias naturales, de tecnologías (mejor si son «nuevas»), y no es extraño que las propuestas de aumento de la presencia horaria de esas disciplinas en el conjunto de los currículos escolares sean bien recibidas. Pero los contenidos y las formas que deben tener esas enseñanzas no suelen ser muy discutidos. Tampoco lo son las razones por las que no siempre les resultan atractivas a los alumnos y por generar en ellos importantes niveles de fracaso. La sociedad demanda de las instituciones educativas una oferta de cultura científica y tecnológica para los nuevos ciudadanos con el convencimiento de que eso es algo importante, pero, una vez hecha esa demanda, no pone condiciones a su cumplimiento, sino que entrega un cheque en blanco a quienes diseñan los currículos tecnocientíficos, considerando que su papel como expertos garantizará que sabrán articular del mejor modo las enseñanzas de las ciencias y las tecnologías (González et al., 1998).

Si se analiza qué están haciendo en otros países para producir reformas educativas, se observa que las políticas adoptadas son bastante más drásticas y de envergadura que en nuestro país, configurando un nuevo paradigma. 


\section{4 \\ ANA ISABEL PARAMÁ, ISABEL CABALLERO, JUAN R. COCA Y JUAN MILTON JAIR ARAGÓN IMPACTO PSICO-SOCIO-EDUCATIVO DE LA TECNOCIENCIA EN UNA HUMANIDAD GLOBALIZADA}

Estados Unidos (EE. UU.) ha fijado políticas gubernamentales para la educación en ciencias, tecnología, ingeniería y matemática (STEM) (GAO, 2005, 2006, 2012, 2014). Prácticamente todas las universidades americanas han organizado Institutos o Centros STEM. El objetivo es cumplimentar una participación fundamental en la educación formal básica preuniversitaria de 13 años (K-12) y una educación formal universitaria y postuniversitaria de siete años más en el área STEM, citamos algunas de estas universidades a modo de ejemplo (Neu, 2016; Umich, 2016; UVG, 2016).

Por su parte, la Unión Europea ha desarrollado el proyecto «Developing Quality in Mathematics Education", en el cual se han elaborado diversos materiales de acuerdo con las demandas actuales sobre educación matemática; citamos a modo de ejemplo algunos de los resultados obtenidos (Andersson, Lingefjärd, Meier y Müller, 2009; Benacka, 2016; Henn, 2009; Lingefjärd y Meier, 2011, 2010; Szilárd y Örs, 2010). También ha implantado, entre otros, el Proyecto REMATH (Representaciones Matemáticas con Medios Digitales), en el que colaboran universidades de París, Londres, Siena, Atenas y centros tecnológicos, para desarrollar dispositivos para la enseñanza de matemática en la era de la computación (Remath, 2016). El Proyecto PRIMAS (Promoting Inquiry in Mathematics and Science Education Across Europe) aspira a cambiar la forma de enseñar ciencias y matemática introduciendo nueva pedagogía, proporcionando a docentes materiales de enseñanza adecuados y el entrenamiento necesario. Hasta el presente se han reunido 14 participantes de 12 países europeos para trabajar juntos y diseminar las estrategias del Proyecto (Primas, 2016). También debemos mencionar la asociación European Schoolnet, que promueve diversos programas relacionados con STEM.

Desde nuestro punto de vista, el propósito de alfabetización científica no debe suponer un descenso del nivel de contenidos para hacer asequible la ciencia a la generalidad de los ciudadanos, sino una reorientación de la enseñanza necesaria para modificar la imagen deformada de la ciencia hoy socialmente aceptada, así como luchar contra los movimientos anticiencia que se derivan, para hacer posible una adquisición significativa de los conceptos. Y aunque es cierto que, como afirma Bybee (1997), la alfabetización científica debe concebirse como un continuo que puede desarrollarse a lo largo de la vida de cada cual y que, por supuesto, unos desarrollarán más que otros, resulta esencial romper con interpretaciones erróneas de lo que supone esa alfabetización y valorar positivamente la orientación multidimensional de la educación científica para todos, incluidos los propios científicos.

Por otro lado, consideramos que los ciudadanos deberían conocer aquello que les permita manejarse y participar en el mundo en el que viven. En la medida en que el desarrollo tecnocientífico ha generado un mundo complejo, pensamos que la educación debería aportar el desarrollo de destrezas y habilidades en los individuos que les permitan manejarse en ese mundo. Por tanto, la enseñanza de las ciencias, sobre todo de las tecnologías, debería hacer posible que los ciudadanos puedan desenvolverse con habilidad en los distintos contextos en los que desarrollan sus vidas, sin la necesidad constante de pedir auxilio a los expertos o de esperar que sean ellos quienes resuelvan los problemas prácticos. Pero manejarse en el 
mundo es saber elegir también la orientación de la propia vivienda, componer una dieta o hacer un itinerario por el bosque otoñal y disfrutar de él. Por último, estamos totalmente de acuerdo con Désautels y Larochelle (2003) en que los modelos discursivos, secuenciales, rígidos, verticales y unidireccionales en la organización de las actividades en el aula y en la sociedad no son los más adecuados para hacer posible la participación de los ciudadanos. Si en la sociedad se dan controversias sobre cuestiones tecnocientíficas que involucran a diversos actores con intereses y valores enfrentados, en las aulas de ciencias y tecnologías también se puede y se debe preparar a los alumnos para la participación en esas controversias y en esas decisiones que les esperan como futuros ciudadanos.

\section{CONCLUSIONES}

El desarrollo de la tecnociencia trae consigo un buen número de soluciones a determinados problemas existentes, pero, al hacerlo, automáticamente genera otros. Ahora bien, el progreso tecnocientífico no puede ser calificado como positivo o negativo de manera esencial unívoca. La actividad tecnocientífica puede traer consigo aspectos positivos o negativos. Ello debe ser tenido en cuenta siempre que se analice dicha actividad. Por lo tanto, creemos que este aspecto no ha sido detectado en ningún otro trabajo previo, la tecnociencia será asumida siempre y cuando facilite nuestro quehacer diario. Si, por el contrario, el desarrollo tecnocientífico genera un conocimiento que trae consigo un incremento de la complejidad de nuestra vida o nuestro trabajo, entonces dicho conocimiento será, en principio, rechazado o postergado.

En este trabajo hemos mostrado una serie de ejemplos, a nuestro juicio, paradigmáticos en los que el desarrollo tecnocientífico implica una gran transformación a nivel psico-socio-educativo. El problema es que materializar este conocimiento supone una gran transformación del proceso educativo actual y convencional. Esto genera cierta complejidad en el sistema, originando que los agentes del mismo se resistan a la adquisición de este conocimiento. En caso contrario, el sistema educativo se vería profundamente mutado. Por ello, podemos afirmar que los elementos arracionales existentes en el sistema pedagógico, pese a las interpenetraciones del sistema tecnocientífico, son relegados a un espacio de parálisis y, en cierto modo, ensombrecidos por el estándar del sistema.

A todo esto, debemos añadir que el análisis histórico permite entender que las elecciones de los científicos y tecnólogos obedecen en menor medida a criterios racionales o fenómenos observacionales propios del sistema. En cambio, deciden atendiendo a razones que pueden ser consideradas al menos como "extracientíficas» o "antilógicas». Siendo en la mayoría de los casos razones de oportunidad, pragmatismo, miedo, poder, económicas, expectativas de éxito o simplemente por gusto las que intervienen en la decisión científica, las cuales son correlatos que no corresponden a la autorreferencia de la tecnociencia. Somos de la opinión de que la ciencia es una actividad que pretende resolver problemas ("problem-solving", 
según la formulación de Larry Laudan, 1997). Es decir, la ciencia se entiende, evalúa y desarrolla en la medida en que resuelve, propone o minimiza problemas. Sin embargo, los problemas le llegan al científico de las tradiciones y valores sociales a los que pertenece, desde su cosmovisión, que incluye los modos de desarrollar, modificar y crear teorías científicas y propuestas tecnológicas.

Los distintos estudios sobre la educación, como ciencias que son, no son ajenos a estos aspectos y necesitan seguir asumiendo un gran número de retos. Las diversas disciplinas tecnocientíficas están ampliando nuestro conocimiento introduciendo mayor información y mayor número de variables en el sistema educativo. Ello supone la generación de una buena cantidad de retos para este sistema. Veremos cómo se gestionará este conocimiento durante los años futuros.

\section{REFERENCIAS BIBLIOGRÁFICAS}

AEPNYA (2008) Protocolos de la Sociedad Española de Psiquiatría Infantil de la AEP. Madrid, AEPNYA.

AiKenHEAD, G. S. (1985) Collective decisión making in the social context of science. Science Education, 69 (4), 453-475.

Alic, M. (1986) El legado de Hipatia. Madrid, Siglo XXI.

Allchin, D. (1998) Values in Science and in Science Education, en Fraser, B. J. y Tobin, K. G. (eds.) International Handbook of Science Education. Dordrecht, Kluwer Academic Publishers, 1083-1092.

Andersson, M.; LingefjÄRd, T.; MeIER, S. y Müller, J. H. (2009) The sun hour project, in Henn, W. y Meier, S. (eds.) Growing mathematics. Dortmund, Print on demand.

BenACKA, J. (2016) Numerical Modelling with Spreadsheets as a Means to Promote stem to High School Students. Eurasia Journal of Mathematics, Science \& Technology Education, 12 (4), 947-964.

Bingle, W. y GaSKell, P. J. (1994) Scientific Literacy for decision making and the social construction of scientific knowledge. Science Education, 78 (2), 185-201.

Briggle, A. y Mitcham, C. (2012) Ethics and Science. An introduction. Cambridge, Cambridge University Press.

Bybee, R. (1997) Towards an Understanding of Scientific Literacy, en Graeber, W. y Bolte, C. (eds.) Scientific Literacy. Kiel, IPN.

Clark, C. N. y Warren, J. D. (2015) Music, memory and mechanisms in Alzheimer's disease brain. A Journal of Neurology, 138, 2122-2125.

CocA, J. R. (2010) La comprensión de la tecnociencia. Huelva, Hergué.

CocA, J. R. (2011) Aplicación de la hermenéutica analógica a la gestión del conocimiento tecnocientífico, en Conde Gaxiola, N. (comp.) Hermenéutica, analogía y sociedad. México, Editorial Torres Asociados, 55-67.

COCA, J. R.; SANTASILIA, S. y VALERO-MATAS, J. (2012) Universalità, globalizzazione e interculturalità. La questione del sistema tecnoscientifico tra sociologia e filosofia sociale. Per la filosofía, 86 (3), 47-62.

CuEvas, A. (2009) Lo incierto, lo racional y lo razonable en el desarrollo de políticas científicas. Revista de Investigaciones Políticas y Sociológicas, 8 (1), 51-63.

DésAutels, J. y LAROCHELle, M. (2003) Educación científica: el regreso del ciudadano y de la ciudadana. Revista Electrónica Enseñanza de las Ciencias, 21 (1), 3-20. 
ANA ISABEL PARAMÁ, ISABEL CABALLERO, JUAN R. COCA Y JUAN MILTON JAIR ARAGÓN

IMPACTO PSICO-SOCIO-EDUCATIVO DE LA TECNOCIENCIA EN UNA HUMANIDAD GLOBALIZADA

Donnison, J. (1977) Midwives and Medical Men: A History of Interprofessional Rivalries and Women's Rights. Londres, Heinemann.

Dumoulin-Bridi, M. C.; Aton, S. J.; Seibt, J.; Renouard, L.; Coleman, T. y Frank, M. G. (2015) Rapid eye movement sleep promotes cortical plasticity in the developing brain. Science Advance. DOI: 10.1126/sciadv.1500105.

ECHEverría, J. (1995) Filosofía de la ciencia. Madrid, Akal.

Echeverría, J. (2002) Ciencia y Valores. Barcelona, Ediciones Destino.

EcheverRía, J. (2003) La revolución tecnocientífica. Madrid, Fondo de Cultura Económica.

Ehrenreich, B. y English, D. (1976) Witches, Midwives and Nurses: A History of Women Healers. Londres, Writers \& Readers.

FENSHAM, P. J. (1988) Approaches to the teaching of sTS in science education. International Journal of Science Education, 10 (4), 346-456.

FENSHAM, P. J. (2004) Beyond knowledge: other scientific qualities as outcomes for school science education, en JANIUK, R. M. y SAMONEK-MiciuK, E. (eds.) Science and technology education for a diverse world: dilemmas, needs and partnerships. Lublin, Maria CurieSklodowska University Press, 23-25.

FEYERABEND, P. (1986) Tratado contra el método. Esquema de una teoría anarquista del conocimiento. Madrid, Tecnos.

GAO (2005) U. S. Government Accountability Office. Federal Science, Technology, Engineering, and Mathematics Programs and Related Trends, GAO-06-114, oct., 2005. Consultado el 1 de septiembre de 2016. http://www.gao.gov/products/GAO-06-114.

GAO (2006) U. S. Government Accountability Office. Higher Education: Science, Technology, Engineering, and Mathematics Trends and the Role of Federal Programs GAO-06-702T, mayo, 2006. Consultado el 1 de septiembre de 2016. http://www.gao.gov/products/ GAO-06-702T.

GAO (2012) U. S. Government Accountability Office. Science, Technology, Engineering, and Mathematics Education: Strategic Planning Needed to Better Manage Overlapping Programs across Multiple Agencies GAO-12-108, enero, 2012. Consultado el 1 de septiembre de 2016. http://www.gao.gov/products/GAO-12-108.

GAO (2014) U. S. Government Accountability Office. Science, Technology, Engineering, and Mathematics Education: Assessing the Relationship between Education and the Workforce GAO-14-374, mayo, 2014. Consultado el 1 de septiembre de 2016. http:// www.gao.gov/products/GAO-14-374.

García Nieto, M. T. (2013) ¿Son invisibles las mujeres científicas? Estudios sobre el Mensaje Periodístico, 19, 783-792.

Gil, D.; Carrascosa, J.; Furió, C. y Martínez-Torregrosa, J. (1991) la enseñanza de las ciencias en la educación secundaria. Barcelona, Horsori.

GonZÁlez-GARCíA, M. I. (1998) Convergencia y conflicto de valores: el caso de las diferencias sexuales en habilidades cognitivas, en AmBrogi, A. (ed.) La naturalización de la filosofía de la ciencia. Palma de Mallorca, Universidad de las Islas Baleares.

González-García, M. I.; López-Cerezo, J. A. y Luján-López, J. L. (1996) Ciencia, Tecnología y Sociedad. Una introducción al estudio social de la ciencia y la tecnología. Barcelona, Tecnos.

GREENFIELD, T. A. (1996) Gender ethnicity, science achievement and attitudes. Journal of Research in Science Teaching, 33, 901-934.

Hare-Mustin, R. T. y MareceK, J. (eds.) (1994) Marcar la diferencia. Psicología y construcción de los sexos. Barcelona, Herder. 
Hendley, D.; Stables, S. y Stables, A. (1995) Gender differences in pupil attitudes to the national curriculum foundation subjects of English, mathematics, science and technology in Key Stage 3 in South Wales. Educational Studies, 21 (1), 85-97.

Henn, H. W. (2009) Warum manchmal Katzen vom Himmel fallen oder: Von guten und von schlechten Modellen, en MEYER, J. y REINEKE, V. (eds.) Schriftenreihe uniKIK. Hannover: Leibniz University, 1-21.

Herrera, R. (1992) Los Sistemas Tecnológicos Concretos. Ingeniería, 2 (2), 41-56.

Herrera, R. (2009) La tecnociencia y la educación: la problemática de su integración. Revista de Filosofía de la Universidad de Costa Rica, XLVII (120-121), 9-17.

Jiménez, M. P. y Álvarez, M. (1992) Género, ciencia y tecnología, en Moreno, M. (ed.) Del silencio a la palabra. Madrid, Instituto de la Mujer, 178-196.

Koelsch, S. (2015) Music-evoked emotions: principles, brain correlates, and implications for therapy. Annals of the New York Academy of Sciences, 1337, 193-201. DOI: 10.1111/ nyas. 12684

LAUDAN, L. (1986) El progreso y sus problemas. Madrid, Encuentro.

LEE, V. E. y BRYK, A. S. (1986) Effects of single-sex secondary schools on student achievement and attitudes. Journal of Educational Psychology, 78 (5), 381-395.

LingEfJÄRD, T. y MeIER, S. (2010) Teachers as managers of the modelling process. Mathematics Education Research Journal, 22 (2), 92-107.

LingefjÄRd, T. y MeIER, S. (2011) The Sun hour project, en KAISER, G.; Blum, W.; BorromeO FERri, R. y STILlman, G. (eds.) Trends in Teaching and Learning of Mathematical Modelling. Heidelberg, Germany, Springer, 97-106.

LiREs, A. M.; NuÑO, T. y SOlSONA, N. (2003) Las científicas y su bistoria en el aula. Madrid, Síntesis.

LOngInO, H. (1990) Science as Social Knowledge: Values and Objectivity in Scientific Inquiry. Princeton, Princeton University Press.

LÓPEZ-NAVAJAS, A. (2014) Análisis de la ausencia de las mujeres en los manuales de la ESO: una genealogía de conocimiento ocultada. Revista de Educación, 363, 282-308.

Luhmann, N. (1992) Sistemas Sociales. Lineamientos para una Teoría General. México DF, Alianza Universidad Iberoamericana.

MaIso, J. (2015) Omnipotencia tecnocientífica y Responsabilidad. La biología sintética en el horizonte de la Bioeconomía, en Actas I Congreso internacional de la Red española de Filosofía, XVI, 93-102.

Manassero-Mas, M. A. y VÁzQuez-Alonso, A. (1996) Factores determinantes de las actitudes relacionadas con la ciencia. Revista Española de Pedagogía, 54 (203), 43-77.

Marcel, G. (2003) Ser y tener. Salamanca, Editorial Caparrós.

Martínez Moscoso, D. M. (2012) Práctica docente con equidad de género. Una guía de trabajo. Guadalajara, Amaya Ediciones.

Morawski, J. G. (1994) Practicing Feminism, Reconstructing Psychology. Ann Arbor, University of Michigan Press.

National Research Council (1996) National Science Education Standards. Washington, D. C., National Academy Press.

NEU (2010) Northeastern University-The Center for STEM Education. Consultado el 10 de agosto de 2016. http://www.stem.neu.edu.

NúÑEz-Jover, J. (2007) La ciencia y la tecnología como procesos sociales. Lo que la educación científica no debería olvidar. La Habana, Editorial Félix Varela. 
ANA ISABEL PARAMÁ, ISABEL CABALLERO, JUAN R. COCA Y JUAN MILTON JAIR ARAGÓN IMPACTO PSICO-SOCIO-EDUCATIVO DE LA TECNOCIENCIA EN UNA HUMANIDAD GLOBALIZADA

OECD (2015) What Lies Behind Gender Inequality in Education? PISA in Focus, 49, OECD Publishing. Consultado el 6 de septiembre de 2016. http://dx.doi.org/10.1787/5js4xffhhc30-en.

Ogilvy, J. A. (2002) Creating Better Futures: Scenario Planning as a Tool for a Better Tomorrow. Oxford, Oxford University Press.

Paramá, A.; Francisco, F. J. y Fernández, C. (2014) Hermenéutica analógica en una realidad social dependiente de la tecnociencia: entre el progreso y la docencia. Revista Inclusiones, 1 (4), 107-117.

PARAmÁ, A. y Rodrigo, P. (2015) Ciencia, sociedad e interculturalidad. 100Cs, 1 (1).

PÉREZ-SEDEÑo, E. (1994) Mujeres matemáticas en la historia de la ciencia, en Matemáticas y coeducación. OECM, Ada Byron.

PÉREZ-SEDEÑO, E. (1998a) Las amistades peligrosas, en GómeZ, A. (ed.) La construcción social de lo femenino. España, Universidad de La Laguna.

PÉREZ-SEDEÑO, E. (1998b) Factores contextuales, tecnología y valores: ¿¿desde la periferia?, en Martínez Freire, P. (ed.) Filosofía actual de la ciencia, suplemento núm. 13 de Contraste.

PRIMAS (2010) Promoting Inquiry in Mathematics Education. 7FP Unión Europea. PDF file. Consultado el 10 de octubre de 2010. http://www.nottingham.ac.uk/education/ documents/research.

Queraltó, R. (2003) Ética, tecnología y valores en la sociedad global. Madrid, Tecnos.

Quintanilla, M. Á. (2005) Tecnología: Un enfoque filosófico. Madrid, Fondo de Cultura Económica.

REMATH (2010) Representing Mathematics with Digital Media. The Amusement Park. Consultado el 1 de septiembre de 2016. http://remath.cti.gr.

RusseT, C. (1989) Sexual Science. The Victorian Construction of Womanhood. Cambridge, Harvard University Press.

SCHIEBINGER, L. (1989) The Mind Has No Sex: Women in the Origins of Modern Science. Cambridge, MA, Harvard University Press.

SJøBERG, S. (2000) Science and scientists: the SAS-study: cross-cultural evidence and perspectives on pulpils' interests, experiences and perceptions - background, development and selected results. Oslo, University of Oslo.

Simpson, R. D. y Oliver, J. E. (1990) A summary of major influences on attitude toward an achievement in science among adolescent students. Science Education, 74 (1), 1-18.

SOlBES, J. y Vilches, A. (1997) STS interactions and the teaching of Physics and Chemistry. Science Education, 81 (4), 337-386.

SOlsOna y PAIRÓ, N. (1997) Mujeres científicas de todos los tiempos. Madrid, Talasa.

SzILÁRD, A. y ÖRS, N. (2010) Measuring with unscaled pots algorithm versus chance. The Electronic Journal of Mathematics and Technology, 4, 1-10.

TRumper, R. (2004) Science and technology education for a diverse world: dilemmas, needs and partnerships. Lublin, Maria Curie-Sklodowska University Press.

Tuana, N. (1993) The Less Noble Sex: Scientific, Religious, and Philosophical Conceptions of Woman's Nature. Bloomington, Indiana University Press.

uMiCH (2016) M-STEM Academies. Consultado el 10 de agosto de 2016. https://mstem.umich. edu/.

Unger, R. y Crawford, M. (1996) Women and Gender. A Feminist Psychology. Nueva York, McGraw-Hill.

UVG (2016) UVG Centro de educación STEM. Consultado el 10 de agosto de 2016. http://uvg. edu.gt/stem/es/stem.html. 
VAllet, M. (2004) Educar a niños y niñas de 0 a 6 años. Barcelona, Cisspraxix.

VÁzQuez-Alonso, A. y MANassero-Mas, M. A. (2003) Las mujeres científicas: un grupo invisible en los libros de texto. Investigación en la Escuela, 50, 31-46.

Velten-Schurian, Y.; Hautzinger, M.; Poets, C. F. y Schlarb, A. A. (2010) Association between sleep patterns and daytime functioning in children with insomnia: The contribution of parent-reported frequency of night waking and wake time after sleep on set. Sleep Medicine, 11 (3), 281-288.

ZabalZa, M. Á. (2010) Didáctica de la Educación Infantil. Madrid, Narcea. 